\title{
Reflexões sobre a Economia Circular
}

\author{
Margarete Blume Vier ${ }^{1}$ \\ Dusan Schreiber ${ }^{2}$ \\ Cristiane Froehlich ${ }^{3}$ \\ Vanusca Dalosto Jahno ${ }^{4}$
}

Submissão: $10 / 03 / 2021$

Aceite: $27 / 07 / 2021$

\begin{abstract}
Resumo
O atual modelo econômico, baseado num modelo linear de produção, resulta, principalmente, no consumo desenfreado da população, na grande geração de resíduos sólidos e na escassez dos recursos naturais. Neste contexto, se faz necessário a adoção do modelo circular de produção, com o objetivo de amenizar os impactos ambientais e alcançar o desenvolvimento sustentável. Nesse contexto, a presente pesquisa tem por objetivo refletir sobre um novo paradigma de sustentabilidade baseado na Economia Circular, que busca não somente benefícios econômicos, mas também, sociais e ambientais. A opção do percurso metodológico foi pela abordagem hermenêutica e método interpretativo, por situar este trabalho dentro do campo das ciências sociais. $O$ trabalho elenca os desafios e os benefícios encontrados na adoção do modelo econômico circular, e ainda, apresenta uma discussão, de diversos autores, em relação a diferentes facilitadores na implantação deste conceito.
\end{abstract}

Palavras-chave: Gestão ambiental. Impactos ambientais. Modelo econômico. Desenvolvimento sustentável.

\section{Reflections on the Circular Economy}

\begin{abstract}
The current economic model, based on a linear production model, results mainly from the unrestrained consumption of the population, through excessive generation of solid waste, thus contributing to the scarcity of natural resources. In this context, it is necessary to adopt the circular model of production, whose objective is to mitigate environmental impacts and achieve sustainable development. Aiming to contribute to the discussion on that topic, this research aims to reflect on a new sustainability paradigm, based on the Circular Economy, which seeks not only economic, but also social and environmental benefits. The methodological path was chosen by the hermeneutic approach and the interpretative method, as it places this work within the social sciences field. The work highlights the challenges and benefits found in the adoption of the circular economic model, and also presents a discussion, by several authors, regarding different facilitators in the implementation of this concept.
\end{abstract}

Keywords: Environmental management. Environmental impacts. Economic model. Sustainable development

\section{Introdução}

A sociedade internaliza os valores que sustentam o paradigma de que para haver desenvolvimento é necessário que haja um crescimento econômico exagerado, o que resulta na exploração descontrolada dos recursos naturais e no consumo exorbitante da população, gerando assim, uma produção desenfreada de resíduos sólidos urbanos, que são descartados em aterros sanitários, ou a céu aberto, de volta ao meio ambiente, causando impactos

\footnotetext{
${ }^{1}$ Mestrado em Qualidade Ambiental (FEEVALE). Servidora Pública na Prefeitura Municipal de Dois Irmãos/RS. Email: marga vier@hotmail.com

2 Doutorado em Administração (UFRGS). Professor do Programa de Pós-Graduação em Qualidade Ambiental da Universidade Feevale (FEEVALE). E-mail: dusan@feevale.br

${ }^{3}$ Doutorado em Administração (UNISINOS). Professora do Programa de Pós-Graduação em Qualidade Ambiental da Universidade Feevale (FEEVALE). E-mail: cfroehlich@feevale.br

${ }^{4}$ Doutorado em Ciências da Saúde (PUC-RS). Professora do Programa de Pós-Graduação em Qualidade Ambiental da Universidade Feevale (FEEVALE). E-mail: vanusca@feevale.br
} 
ambientais (REIS; FADIGAS; CARVALHO, 2012). Esta situação é consequência do atual modelo econômico, baseado no modelo linear de produção, que consiste em extrair, transformar, produzir, utilizar e descartar. Este modelo exterioriza dois graves problemas ambientais da atualidade, que é a geração de resíduos e a escassez de recursos naturais.

Dados divulgados pela Associação Brasileira das Empresas de Limpeza Pública e Resíduos Especiais (ABRELPE, 2019) apontam que no ano de 2018 foram gerados 79 milhões de toneladas de resíduos sólidos urbanos no Brasil. Deste montante, 72,7 milhões foram coletados, os quais 43,3 milhões de toneladas foram destinados à aterros sanitários, e 29,4 milhões foram descartados em "lixões". Ressalta-se que estes dados se referem apenas aos resíduos sólidos urbanos.

De acordo com a Organização Mundial das Nações Unidas (ONU, 2015), em 2013, apenas um quinto dos recursos, utilizados no mundo, foram provenientes de fontes renováveis. Em virtude disso, uma das metas da Agenda de Desenvolvimento Sustentável para 2030 é justamente o uso eficiente dos recursos naturais, devido a voracidade com que se têm consumido estes recursos.

Como alternativa a este modelo linear de produção que tem como consequência, principalmente, a extração intensiva de recursos naturais e a geração excessiva de resíduos, foi concebido o modelo circular de produção. O conceito de Economia Circular visa fechar o ciclo de vida dos produtos, para que, ao final de sua vida útil, os mesmos possam ser reaproveitados, reutilizados ou reciclados, gerando assim, benefícios econômicos, sociais e ambientais.

Diante desse cenário, o presente trabalho tem por objetivo promover uma reflexão acerca da adoção da Economia Circular, seus desafios, benefícios e os pilares que estruturam este novo paradigma de sustentabilidade. A opção do percurso metodológico foi pela abordagem hermenêutica e o método interpretativo.

\section{Procedimentos metodológicos}

Este trabalho foi construído com o intuito de refletir sobre um novo paradigma de sustentabilidade, baseado na Economia Circular, que busca não somente benefícios econômicos, mas também, sociais e ambientais. A escassez dos recursos naturais e a geração de resíduos impactam diretamente o ambiente interno e externo das organizações e da sociedade de forma geral. Sendo assim, a reflexão acerca deste novo modelo econômico inclui as mudanças culturais e o impacto delas sobre as organizações, assim como a importância de 
mudar a forma com que as empresas produzem e prestam seus serviços, e os benefícios gerados por este novo modelo econômico.

Em relação ao desenvolvimento sustentável, se faz necessário uma visão sistêmica sobre os negócios e as externalidades destes na sociedade e o meio ambiente. Esta visão contraria o pressuposto capitalista ainda presente nas organizações e considerado normal pelos consumidores, o que evidencia o desafio existente na implantação deste novo paradigma. No entanto, este trabalho apresenta a discussão de diversos autores em relação a diferentes facilitadores na implantação do modelo econômico circular.

A opção do percurso metodológico foi pela abordagem hermenêutica e método interpretativo, por situar este trabalho dentro do campo das ciências sociais, de acordo com a proposta de investigação apresentada. A hermenêutica, como abordagem de investigação científica, foi concebida, originalmente, para interpretar os textos sagrados, clássicos e jurídicos, com o intuito de promover uma reflexão teórico metodológica (DOMINGUES, 2004). O seu surgimento é atribuído ao filósofo Wilhelm Dilthey, no ano 1900, com aportes teóricos relevantes de autores como Weber (1979), Mannheim (1974), Heidegger (1999), Hekman (1990) e Habermas (2000). A abordagem hermenêutica é considerada como mais adequada para a análise de fenômenos sociais e culturais e consiste na interpretação das visões de mundo, em determinados recortes temporais, históricos e contextuais (WEBER, 1979; MANNHEIM, 1974; HEIDEGGER, 1999; HEKMAN, 1990; HABERMAS, 2000; DOMINGUES, 2004).

O eixo estruturante deste trabalho consiste na identificação de aspectos centrais que são discutidos por diferentes autores, promovendo o diálogo entre eles acerca do conceito, dos desafios e benefícios na adoção deste modelo econômicos, e ainda, os pilares que sustentam a Economia Circular. A escolha dos autores selecionados para participar do debate ocorreu com base no critério da contribuição oferecida em seus respectivos campos de investigação. A revisão bibliográfica foi realizada por meio de consultas em bancos de dados como Capes, Scielo, Scopus e Unique, selecionando-se os artigos com maior relevância e aderência ao tema estudado, além dos artigos citados por outros autores. A busca inicial ocorreu por meio da palavra-chave principal "economia circular" e "circular economy". A partir da leitura dos primeiros artigos, identificaram-se outras palavras chaves que eram consideradas pilares na Economia Circular; como por exemplo, "simbiose industrial”, "ecodesign", e, "avaliação do ciclo de vida"; além disso, utilizou-se a combinação de palavras, como: "modelo de negócio" e "economia circular", "economia circular" e "sistema de gestão ambiental”. 
A partir destas outras palavras-chave, ampliou-se a busca por artigos científicos, sendo feita, primeiramente, a leitura do resumo, onde era verificado se o artigo tinha aderência, ou não, ao tema estudado. Após a primeira triagem, era feita a leitura completa do artigo, juntamente com fichas resumo, contendo os principais tópicos tratados. Através da leitura completa dos artigos, identificaram-se diversos outros artigos que foram mencionados pelos autores, e que eram relevantes para o estudo; e, portanto, foram incluídos na revisão bibliográfica. Este processo ocorreu durante o ano de 2018 e no primeiro trimestre de 2019, sendo que neste período foram analisadas torno de 125 produções científicas, entre artigos, dissertações, teses de doutorado e livros. Os textos foram lidos na íntegra e submetidos à análise interpretativa dos autores da pesquisa, considerando as recomendações da abordagem hermenêutica qualitativa, procurando situá-los contextualmente e historicamente, facultando a identificação de aspectos que podem respaldar a hipótese estruturante deste trabalho, que é a recomendação da adoção da Economia Circular como um novo modelo econômico.

\section{Surgimento de um novo paradigma em sustentabilidade: Economia Circular}

Historicamente, o homem sempre explorou os recursos naturais sem se preocupar com os danos causados ao meio ambiente (ALBUQUERQUE et al., 2009). O crescimento econômico foi baseado num modelo linear de produção, que consiste em extrair, transformar, produzir, utilizar e descartar (ELLEN MACARTHUR FOUNDATION, 2015). A adoção do modelo linear de produção, principalmente após a Segunda Guerra Mundial, foi relevante para o desenvolvimento industrial, pois os recursos naturais eram considerados abundantes e ilimitados na natureza, o que os tornava uma fonte de matéria prima de baixo custo e de fácil acesso (ELLEN MACARTHUR FOUNDATION, 2015). Entretanto, impulsionou também o uso descontrolado dos recursos naturais e a geração de grandes quantidades de resíduos (LEITÃO, 2015; RAZERA et al., 2017).

A maioria dos impactos ambientais é decorrente da aceleração do desenvolvimento com ênfase apenas no resultado econômico (crescimento econômico), sem o controle e a preocupação com a preservação ambiental (ALBUQUERQUE et al., 2009). Em função disso, Ellen MacArthur Foundation (2015) considera o modelo linear de produção como sendo um modelo insustentável, e que causa graves impactos ambientais, principalmente porque este modelo visa o desenvolvimento industrial, sem considerar os aspectos sociais e ambientais. 
Além disso, a dependência deste modelo de produção por recursos naturais não renováveis, os quais tornam-se cada vez mais escassos, causa significativas alterações nos preços das commodities (LEITÃO, 2015; SACIROVIC; KETIN; VIGNJEVIC, 2018), causando uma instabilidade econômica. O aumento da população e do consumo nos países em desenvolvimento, aumentam ainda mais os preços das matérias primas, e, consequentemente, os custos para as empresas e consumidores (HOUSE OF COMMONS, 2014). Desta forma, pode se afirmar que este modelo afeta diretamente a sustentabilidade ambiental, considerando que esta representa a ideia de manter os estoques da natureza para gerações futuras, ou a garantia da reposição dos mesmos (MOURA, 2006; MILLER; SPOOLMAN, 2012).

Deste modo, devido à disponibilidade limitada de recursos naturais e a capacidade do planeta de assimilar a poluição gerada, o modelo linear de produção está fortemente comprometido. Se faz necessário um novo modelo de desenvolvimento econômico, um modelo que gere impactos positivos não só na economia, mas também em termos ambientais e sociais. O crescimento econômico e o desenvolvimento tecnológico não precisam ser sinônimos de degradação ambiental (LEITÃO, 2015; MOSTAGHEL; OGHAZI, 2018).

Garcia e Naime (2004) enfatizam a necessidade de conciliar o desenvolvimento econômico e a preservação ambiental através do desenvolvimento sustentável. Eles definem desenvolvimento sustentável como a busca do equilíbrio entre a tecnologia e o ambiente, com a participação de diversos grupos sociais, todos à procura por qualidade de vida, equidade e justiça social. Da mesma forma, Reis, Fadigas e Carvalho (2012) tratam o desenvolvimento sustentável como um meio para se superar os atuais problemas ambientais e sociais; os desastres ecológicos e a existência de grandes populações que vivem em condições de pobreza, além da má distribuição da riqueza natural e humana. Porém, segundo os autores, tais objetivos implicam em mudanças na organização da sociedade humana, no uso dos recursos naturais e nos sistemas de produção.

Neste contexto, surgiu a Economia Circular, com o objetivo de oferecer uma alternativa ao modelo Linear de Produção, e estabelecer um novo paradigma de sustentabilidade. Segundo Korhonen, Honkasalo e Seppala (2017) e Feldmann et al. (2018), a Economia Circular causa impactos positivos no meio ambiente, bem como, na economia; e, portanto, seria uma abordagem para o crescimento econômico em consonância com o desenvolvimento ambiental e o desenvolvimento econômico sustentável.

A Economia Circular é considerada uma fonte de inovação, que visa reduzir a utilização de recursos naturais, com a diminuição dos desperdícios e o reaproveitamento dos resíduos 
(LEITÃO, 2015). A eliminação dos desperdícios na cadeia industrial resulta na economia dos custos de produção e na menor dependência dos recursos naturais (ELLEN MACARTHUR FOUNDATION, 2012). A implantação da Economia Circular envolve três aspectos importantes: a redução do impacto ambiental, os benefícios econômicos e a solução para amenizar a escassez de recursos naturais (MOSTAGHEL; OGHAZI, 2018).

Em relação ao conceito de Economia Circular, este é baseado no funcionamento da própria natureza, na qual não há a geração de resíduos. Sendo assim, é um modelo circular de produção, onde os recursos são repetidamente utilizados, reduzindo o consumo de matéria prima e a geração de resíduos (LEITÃO, 2015; HOUSE OF COMMONS, 2014). A Economia Circular está voltada ao desenvolvimento sustentável, que por meio da inovação e do design, visa "fechar o ciclo de vida" dos produtos e aumentar a eficiência no uso dos recursos naturais, gerando benefícios econômicos, sociais e ambientais (LEITÃO, 2015; GHISELLINI; CIALANI; ULGIATI, 2016; KORHONEN; HONKASALO; SEPPÄLÄ, 2017).

Nesta perspectiva, os produtos são desenvolvidos para que ao final do primeiro ciclo, eles possam ser utilizados como matéria prima para um novo produto, iniciando um novo ciclo. Deste modo, os recursos naturais, hora limitados, são reaproveitados, agregando valor econômico, e diminuindo a necessidade de extração dos recursos na natureza (STAHEL, 2016). Mostaghel e Oghazi (2018) enfatizam ainda a importância de cada um dos envolvidos no ciclo de vida dos produtos, pois se um deles não adotar o modelo circular, este produto encerra o seu ciclo, tornando-se apenas resíduo descartado.

A Economia Circular segue a filosofia "cradle to cradle" (do berço ao berço), onde os resíduos são considerados matéria prima, e ao final de seu ciclo, retornam à natureza; enquanto o modelo linear possui uma abordagem do tipo "cradle to grave" (do berço ao túmulo), e neste caso, os produtos, após o consumo, são descartados, na sua grande maioria, em aterros sanitários (ELLEN MACARTHUR FOUNDATION, 2012; LEITÃO, 2015; ALIGLERI; ALIGLERI; KRUGLIANSKAS, 2016). A Figura 1 apresenta de forma simplificada os modelos linear e circular de produção.

Observa-se que a Economia Circular é representada em forma de círculos. A Ellen MacArthur Foundation (2012) cita que quanto mais apertado for este círculo, melhor. Ou seja, quanto menor for a modificação do produto para ser introduzido novamente na cadeia produtiva, maior o potencial de ganho econômico e energético. Korhonen, Honkasalo e Seppälä, (2017) corroboram que o tempo no qual os produtos são submetidos nos círculos internos 
(reutilização, renovação e reparação), deve ser maximizado, exigindo menos recursos e energia, além de ser uma reciclagem mais econômica.

Figura 1 - Modelo Linear e Modelo Circular de Produção
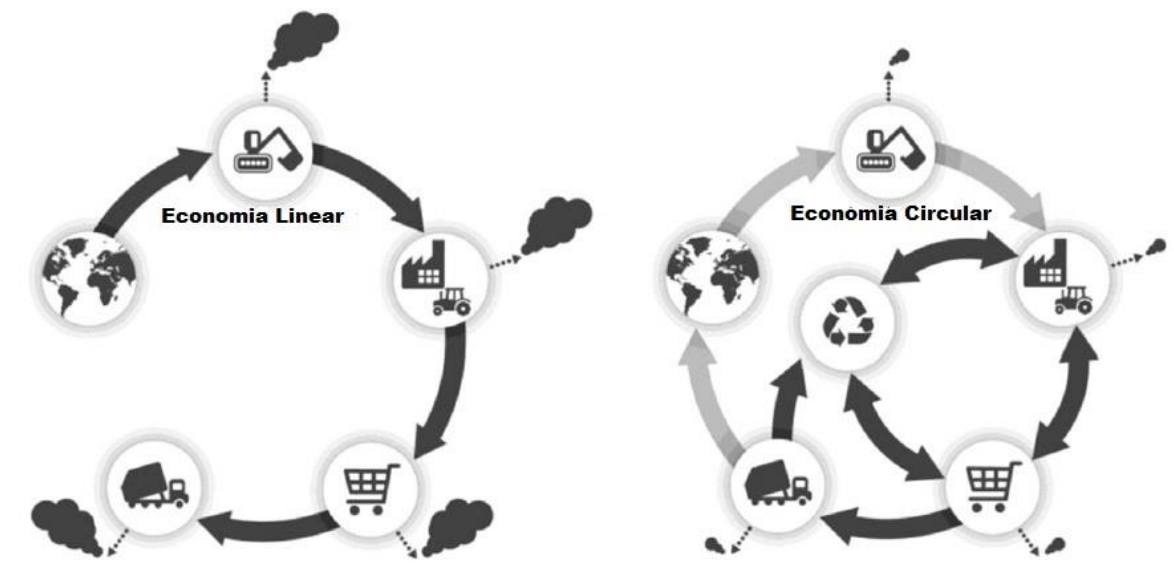

Fonte: Adaptado de Sauvé, Bernard e Sloan (2016, p.5)

Em relação a origem do conceito de Economia Circular, Botezat et al. (2018), Leitão (2015) e Rios e Charnley (2016) afirmam que o atual conceito é, na verdade, uma evolução de outros conceitos. De acordo com os autores, o conceito passou por três estágios: o primeiro foi o "projeto regenerativo" proposto por John T. Lyle na década de 1970; em seguida, a "economia de performance", proposta por Walter Stahel em 1997, a qual introduz a ideia do fechamento do ciclo. A economia de performance, por sua vez, originou o termo "cradle to cradle", que ficou conhecido, principalmente, após o alemão Michael Braungart e o americano William McDonough escreverem o livro "Cradle-to-Cradle: Remaking the way we make things", no ano de 2002.

Entretanto, a Economia Circular continua sendo considerada uma economia regenerativa, e utiliza o termo "cradle to cradle" para explicar a sua filosofia. Desse modo, o que se pode concluir da literatura é que a origem do conceito de Economia Circular não é uma evolução conceitual, e que, de acordo com outros autores (CNI, 2018; ELLEN MACARTHUR FOUNDATION, 2015) a origem do conceito está ligada a diferentes escolas de pensamento, que juntas, formam o atual conceito de Economia Circular. Entre essas escolas, os autores citam o design regenerativo, a ecologia industrial, a biomimética, a própria economia de performance e o cradle to cradle. 


\section{Os ganhos potenciais decorrentes da adoção da Economia Circular nas organizações}

A Ellen MacArthur Foundation (2015) cita benefícios ambientais, econômicos, sociais, operacionais e estratégicos, que atingem não apenas a indústria, mas também o consumidor e a sociedade. De forma geral, pode se afirmar que a Economia Circular é um "modelo que otimiza o fluxo de bens, maximizando o aproveitamento dos recursos naturais e minimizando a produção de resíduos" (LEITÃO, 2015).

Em relação aos benefícios econômicos, percebe-se a redução do custo da matéria prima e da energia, além da possibilidade de geração de renda, com os resíduos antes descartados. Além disso, a Economia Circular pode contribuir para a redução do custo com o descarte de resíduos e com os riscos ambientais. Cita-se ainda os benefícios macroeconômicos, entre eles, a geração de emprego, a redução dos custos com aterros sanitários e uma economia mais estável e resiliente (KORHONEN; HONKASALO; SEPPÄLÄ, 2017; ELLEN MACARTHUR FOUNDATION, 2015; LEITÃO, 2015).

Para as empresas, a Economia Circular proporciona a oportunidade de novos produtos e empreendimentos, ou ainda, novos modelos de negócios (KORHONEN; HONKASALO; SEPPÄLÄ, 2017), além de uma imagem mais sustentável, que pode ser favorável ao marketing da empresa (LEITÃO, 2015; KORHONEN; HONKASALO; SEPPÄLÄ, 2017). Evidentemente que os benefícios econômicos de redução no custo da matéria prima e da destinação de resíduos repercutem nos resultados econômicos das empresas.

Outro benefício que pode ser descrito é a melhora no relacionamento com o cliente, o aumento da confiança e da lealdade, uma vez que se tem uma maior interação entre clientes e fornecedores, por exigir que o produto retorne ao fornecedor, ou então, quando, ao invés de se ter a venda do produto, tem-se a economia compartilhada, exigindo o contato permanente entre os atores envolvidos. Na economia compartilhada, que será estudada mais adiante, ocorre a prestação de serviço de fornecimento de um determinado bem, sendo que neste caso, o consumidor torna-se um usuário do produto (ELLEN MACARTHUR FOUNDATION, 2012).

A Economia Circular reduz ainda a exposição das empresas a preços cada vez mais voláteis, uma vez que se tem um menor uso de materiais virgens e maior consumo de materiais reciclados. Há também uma cultura organizacional mais positiva, decorrente da melhoria nas condições da empresa, colaboradores e parceiros. Com isso, acredita-se que a Economia Circular proporciona às empresas uma maior sustentabilidade para os negócios a médio e longo prazo (ELLEN MACARTHUR FOUNDATION, 2015). 
Os benefícios da Economia Circular para os clientes vão além do preço, pois incluem também a melhora na qualidade do produto, principalmente quando se tem uma economia compartilhada, onde o fornecedor prioriza a durabilidade de seu material. Além disso, os produtos não são mais projetados para serem descartados rapidamente, reduzindo o custo da obsolescência programada para o cliente ou usuário (ELLEN MACARTHUR FOUNDATION 2012, 2015; SPOSATO et al., 2017).

Os benefícios ambientais, além da redução do consumo de recursos naturais, incluem também a redução das emissões de dióxido de carbono. Segundo a Ellen Macarthur Foundation (2015), na Europa, o desenvolvimento da Economia Circular poderia, até 2030, reduzir pela metade os níveis atuais (2015) de emissão de dióxido de carbono na atmosfera. A Economia Circular poderia também melhorar a biodiversidade, a produtividade da terra e a saúde do solo. O maior uso de materiais biológicos, os quais retornam ao solo por meio da digestão anaeróbica ou pelo processo de compostagem, reduz a necessidade de fertilizantes químicos. E por fim, outro benefício ambiental é a redução das externalidades negativas, entre elas, a poluição do ar, da água, a liberação de substâncias tóxicas e as mudanças climáticas (ELLEN MACARTHUR FOUNDATION, 2015).

\section{Os desafios e obstáculos para a aplicação dos conceitos da Economia Circular}

Apesar dos benefícios proporcionados pela Economia Circular, sua adoção torna-se complexa, pois considera diversos fatores; o design de produtos, a distribuição de material e energia, o modelo de negócio, além de exigir grandes mudanças, tanto na sociedade, quanto no interior das grandes empresas. A introdução do conceito nas indústrias enfrenta barreiras financeiras, de estrutura, operacionais, comportamentais e tecnológicas (RITZEN; SANDSTROM, 2017; SMOL; KULCZYCKA; AVDIUSHCHENKO, 2017).

A Economia Circular exige uma reestruturação no modelo de negócio e, na maioria das vezes, na estrutura física da empresa (LEITÃO, 2015). Aligleri, Aligleri e Kruglianskas (2016) afirmam que a Economia Circular desafia as empresas a repensarem os processos, definirem novas tecnologias, reavaliarem a pré-produção, o desenvolvimento dos produtos, os materiais empregados, a sua distribuição, sua funcionalidade, seu descarte; e ainda, a buscarem estratégias afins de desenvolverem ações ambientalmente sustentáveis, socialmente corretas, e economicamente viáveis. 
Reestruturar o modelo de negócio inclui também decisões estratégicas em relação ao papel que as empresas desempenham em uma cadeia de valor, e como se posicionam em relação as demais organizações, ou ainda, como criam valor ao produto e vendem os resultados para o mercado (CNI, 2018; RITZEN; SANDSTROM, 2017). Portanto, adotar um sistema econômico circular exige das empresas mudanças profundas, o que impacta nas decisões dos gestores, e consequentemente, em outro desafio encontrado na literatura, que é a cultura organizacional (BALACEANU; TILEA; PENU, 2017).

A cultura organizacional e a resistência dos gestores às mudanças são barreiras significativas, pois podem inviabilizar qualquer tipo de iniciativa de promover ajustes necessários na organização, especialmente quando estes ajustes representam mudanças relevantes e de caráter inovador (RITZEN; SANDSTROM, 2017; MOSTAGHEL; OGHAZI; 2018). Botezat et al. (2018) destacam que toda a mudança depende de uma tomada de decisão e uma estrutura organizacional interna que apoie as iniciativas.

A referida resistência dos gestores é decorrente do medo do desconhecido, principalmente porque a transição envolve riscos em função das mudanças estruturais, de processo e na relação com os clientes e fornecedores. $O$ aumento da dependência entre os parceiros também pode gerar grandes riscos para as empresas, principalmente por ser um fator externo, que não pode ser controlado por elas (RITZEN; SANDSTROM, 2017; MOSTAGHEL; OGHAZI, 2018). Ressalta-se que a Economia Circular deve estar nos objetivos da empresa e ser difundida entre os funcionários. É essencial que todos os setores adotem este conceito, e o mais importante, que todos os setores estejam interligados, havendo uma cooperação entre eles (LEITÃO, 2015).

No tocante às barreiras financeiras, estas são relatadas por diversos autores (MATIVENGA et al., 2016; MOSTAGHEL; OGHAZI, 2018). Ritzen e Sandstrom (2017) afirmam que as empresas apresentam certa resistência em investir na transição para a Economia Circular, pois consideram o retorno incerto e, principalmente, por este retorno não ser de imediato. Logo, as barreiras financeiras incluem a falta de recursos financeiros para o investimento em si; como também, a resistência das empresas em não quererem investir em um modelo de negócio pouco convencional.

O estudo de Kirchherr et al. (2018) aponta que a maioria das empresas consideram que, a adoção de uma Economia Circular exige um custo inicial muito elevado, e que os modelos de negócio circulares ainda estão sendo aperfeiçoados, e, portanto, as primeiras empresas que forem investir na transição, não terão o retorno esperado. Kirchherr et al (2018) 
afirmam ainda que os elevados custos iniciais podem estar correlacionados com a resistência dos gestores às mudanças, uma das barreiras significativas citada anteriormente.

Para as pequenas e médias empresas, cuja visão é imediatista, o alto custo de investimento, com o retorno no longo prazo, torna-se um grande entrave. Isto porque, o lapso temporal entre o investimento e o retorno é grande, e estas pequenas e médias empresas nem sempre possuem um planejamento de longo prazo, ou uma saúde financeira que possibilite um retorno que não seja imediato (MARTINS; ESCRIVÃO, 2010; PAZ; KIPPER, 2016).

Outra dificuldade encontrada na adoção da Economia Circular, principalmente nas pequenas e médias empresas, é a falta de conhecimento dos gestores sobre os aspectos ambientais, a insuficiência de recursos humanos qualificados e a dificuldade de associar os danos ambientais aos processos realizados pelas empresas. Cita-se ainda, a falta de registros de informações que impactam o meio ambiente, e dos procedimentos realizados dentro das empresas (MARTINS; ESCRIVÃO, 2010; SILVEIRA; ALVES, 2012; MATIVENGA et al., 2016).

Quanto as barreiras tecnológicas, alguns autores relatam a necessidade de investimentos no desenvolvimento de tecnologias, principalmente na recuperação de materiais já utilizados (MOSTAGHEL; OGHAZI, 2018, SMOL; KULCZYCKA; AVDIUSHCHENKO, 2017). Para Leitão (2015), as tecnologias que visam mitigar o impacto ambiental no meio ambiente existem, porém, os processos produtivos ainda estão voltados para a economia linear, dificultando, assim, a adoção das referidas inovações tecnológicas. Corroborando, Kirchherr et al. (2018) afirmam que as principais barreiras não são tecnológicas, mas sim, culturais e de sensibilização da população.

Além das barreiras internas enfrentadas pelas empresas, outro grande problema que impacta diretamente na eficácia da Economia Circular é a cultura de consumo atual. Se a sociedade não tiver um consumo mais consciente, a Economia Circular será apenas mais um conceito fracassado em termos de economia e desenvolvimento sustentável (KORHONEN; HONKASALO; SEPPÄLÄ, 2017; RAZERA et al., 2017).

A conscientização e sensibilização da sociedade quanto à correta separação e destinação dos resíduos, representam outro desafio para a Economia Circular. O descarte incorreto inviabiliza a reutilização, recuperação ou ainda, a reciclagem do material. Além disso, o próprio descarte dos resíduos também é um grande problema, pois, ainda que exista uma legislação contemplando a responsabilidade compartilhada, não é o que acontece na prática. Velis e Vrancken (2015) destacam que o resíduo, uma vez descartado em local público por seu proprietário, é de todos e de ninguém ao mesmo tempo. Em função da limpeza e da saúde 
pública, a responsabilidade deste resíduo recai sobre o poder público, impossibilitando um sistema de logística reversa e fazendo com que as empresas deixam de se preocupar com o resíduo gerado de seus produtos.

O comportamento do consumidor também possui grandes impactos na implantação de uma Economia Circular. Muitos consumidores não valorizam ações sustentáveis, e optam sempre pelo produto de menor preço, mesmo que este tenha um impacto ambiental maior. Ressalta-se ainda a percepção do consumidor em relação à qualidade do produto remanufaturado ou reciclado (WASTLING; CHARNLEY; MORENO, 2018; KIRCHHERR et. al., 2018). O estudo de Mostaghel e Oghazi (2018) aponta ainda que os clientes e usuários, por não valorizarem as ações sustentáveis, não devolvem os produtos aos fornecedores, encerrando assim o ciclo deste material.

Entre os fatores que dificultam a implantação de uma Economia Circular, cita-se ainda as barreiras de mercado. Dentre estas, cita-se a falta de financiamentos, ou ainda, a criação de incentivos fiscais na adoção do sistema, como por exemplo, na utilização de materiais recicláveis, ou na aquisição de tecnologias ambientais. A matéria prima virgem muito barata também contribui para que as empresas não utilizem materiais reciclados ou reutilizados. Ressalta-se ainda que muitos resíduos não são reaproveitados pois a reciclagem torna-se inviável economicamente, e nestes casos, o aterro sanitário é considerado uma melhor opção pelas empresas (STAHEL, 2016; KIRCHHERR et al., 2018; BOTEZAT et al., 2018).

\section{Os pilares da Economia Circular}

Analisando o conceito, os objetivos e os princípios da Economia Circular, e ainda, os benefícios e as barreiras encontradas na adoção deste modelo, observa-se que a Economia Circular envolve inúmeros fatores internos e externos às organizações; e, é baseada em diversos outros conceitos que contribuem para alcançar seus objetivos. Portanto, a Economia Circular é constituída por diversos pilares, ou ainda, fatores essenciais para o adequado funcionamento deste novo modelo econômico, e que se relacionam de alguma forma.

Na implantação de uma Economia Circular, sugere-se a adoção e operacionalização de modelos de negócios sustentáveis, os quais, incorporam os princípios e objetivos da Economia Circular. O modelo de negócio é o conjunto de estratégias e decisões que irão definir a maneira com que as empresas criam, transferem ou capturam valor ao produto ou serviço ofertado, de acordo com as atividades internas e as relações estabelecidas com as partes interessadas 
(URBINATI; CHIARONI; CHIESA, 2017; BOCKEN et al., 2013; CNI, 2018). Existem vários modelos de negócio circulares que podem servir de exemplo, e que empregam diferentes tipos de inovação, entre eles, o que oferece produtos como serviços (PSS), o compartilhamento, os insumos circulares, a extensão da vida do produto e a virtualização (CNI, 2018).

O design do produto ameniza as barreiras na transição para uma Economia Circular, pois, para que o produto possa fechar o ciclo e voltar à cadeia produtiva, é necessário que ele seja projetado para este fim (ALVES; FREITAS, 2013; ELLEN MACARTHUR FOUNDATION, 2015). O ecodesign visa utilizar matérias primas que sejam recicláveis ou reaproveitáveis. É a arte de planejar um produto e já pensar no seu ciclo de vida, na forma com que ele será descartado ou reaproveitado no futuro (NAIME; GARCIA, 2004). Para que se tenha melhores resultados, o ecodesign deve ser aplicado juntamente com a Avaliação do Ciclo de Vida (ACV). A ACV é uma ferramenta para avaliar os impactos ambientais e as consequências à saúde humana associadas a um produto, serviço, processo ou material, ao longo de todo o seu ciclo de vida (SILVA et al., 2012; ASSIS, 2009).

A implantação de uma Economia Circular requer uma cultura organizacional que apoie as ações sustentáveis; o que torna possível a implantação de um Sistema de Gestão Ambiental. (KIRCHHERR et al., 2018; LEITÃO, 2015; RITZEN; SANDSTROM, 2017; MOSTAGHEL; OGHAZI, 2018). De acordo com a ISO 14001 (ISO 14001, 2004) o Sistema de Gestão Ambiental (SGA) é um conjunto de atividades administrativas e operacionais que tem por objetivo solucionar ou amenizar os impactos ambientais, e ainda, evitar o seu surgimento.

A conscientização/sensibilização por parte dos funcionários em relação às questões ambientais, também é muito importante. Os funcionários devem ter conhecimento da importância de seguir a política ambiental da empresa e o sistema de gestão ambiental, estar cientes dos impactos causados pelas atividades que eles exercem, e dos benefícios que tem a observância das regras ambientais para a empresa (MOURA, 2011; SHIGUNOV NETO; CAMPOS; SHIGUNOV, 2009; DIAS, 2011).

Outro fator que contribui para a Economia Circular, e que inclusive é citado por alguns autores, como sendo um dos princípios deste modelo (GORECKI et al., 2018; SANDOVAL; ORMAZABAL; JACA, 2017), é a prática dos 3R: reduzir, reutilizar e reciclar. De acordo com Naime (2005), mesmo com a prática dos 3R, sempre haverá a geração de resíduos, e por isso, se faz necessário a melhor gestão possível, por meio da elaboração adequada de procedimentos e normatizações para gerenciamento dos resíduos. Para a melhor gestão dos resíduos, é importante a elaboração de um Sistema Gerenciador Interno dos Resíduos, que 
conterá a determinação dos procedimentos, os setores responsáveis, as metodologias de segregação na origem, as formas de acondicionamento e transporte, além de planilhas trimestrais de controle de resíduos (NAIME, 2005). Mais importante que a gestão de resíduos é a correta gestão de recursos. Em uma Economia Circular, é muito importante analisar os recursos utilizados nos processos produtivos, e as possibilidades de empregar recursos alternativos, otimizando assim a matéria prima (SANCHEZ et al., 2018; TANTAU; MAASSEN; FRATILA, 2018; VALENTURF et al., 2018).

Outra ferramenta de gestão ambiental, e que pode ser considerada como sendo um dos pilares que contribuem para a adoção de uma Economia Circular, é a Produção mais Limpa $(P+L)$. Esta ferramenta busca o aumento da eficiência, o uso sustentável dos recursos naturais, a redução dos custos e dos desperdícios, a redução dos riscos à sociedade e ao meio ambiente; objetivando assim, o aumento do potencial competitivo das organizações (ALVES; FREITAS, 2013). A P+L busca ainda maximizar a eficiência energética e o uso de energias renováveis, um dos princípios da Economia Circular (GNONI et al., 2017). As melhorias contínuas devem ocorrer também no sistema de logística da empresa, buscando práticas sustentáveis em todo o processo, objetivando a logística verde (ENGELAGE; BORGERT; SOUZA, 2016).

A logística reversa é considerada uma das principais práticas de sustentabilidade das atividades de logística, e é parte integrante de uma abordagem maior, que é a logística verde (ENGELAGE; BORGERT; SOUZA, 2016). Segundo a Ellen MacArthur Foundation (2015) o desenvolvimento do produto pelo fabricante, considerando a sua reutilização após o uso, não terá importância se este material não retornar à sua cadeia produtiva, e por isso, o ciclo reverso é tão importante quanto o design do produto, o modelo de negócio e os demais pilares da Economia Circular.

As externalidades ambientais e sociais devem ser internalizadas como sendo custos ou benefícios econômicos. Sendo assim, se faz necessário que as empresas identifiquem as externalidades negativas, com o objetivo de internalizar a problemática, na busca de uma solução; ou seja, reverter o impacto negativo em um benefício para a empresa, ou então, amenizar este impacto de alguma forma (VELENTURF et al., 2018).

Ressalta-se ainda que as ações sustentáveis podem ser praticadas em conjunto pelas empresas, tendo-se um resultado mais eficaz (GIANETTI; ALMEIDA; BONILLA, 2003). Esta parceria, entre várias organizações interligadas, é denominada Simbiose Industrial, parte integrante da Ecologia Industrial. No conceito de Ecologia Industrial, as indústrias devem operar de forma semelhante aos ecossistemas naturais, interagindo entre si, promovendo parcerias, 
como numa comunidade (GIANETTI; ALMEIDA; BONILLA, 2003; LEITÃO, 2015; SACIROVIC; KETIN; VIGNJEVIC, 2018). Segundo Chertow (2000), a Simbiose Industrial envolve diferentes empresas em uma abordagem coletiva de vantagem competitiva, envolvendo a troca de materiais, subprodutos e energia.

O poder público pode contribuir para a implantação da Economia Circular de diferentes maneiras, através da incentivos e sanções fiscais, por meio do apoio ao modelo econômico circular, com financiamentos, ou ainda, promovendo campanhas educativas. Em um mercado cada vez mais competitivo, as empresas, muitas vezes, optam pela lucratividade, desconsiderando os aspectos sociais e ambientais. Sendo assim, se faz necessário que o governo interfira neste ciclo e promova uma política fiscal, através do incentivo ao uso de material reciclado ou energia renovável, ou então, sanções e aumento de impostos, para produtos que não atendem os princípios circulares. (BALACEANU; TILEA; PENU, 2017; MURAKAMI et al., 2014).

O fator educação compreende a educação dos futuros profissionais, como também da sociedade. A transição para uma Economia Circular requer o envolvimento de todos os atores na sociedade (GHISELLINI; CIALANI; ULGIATI, 2016). São necessários estratégias, políticas públicas e o envolvimento dos profissionais da educação, para sensibilizar a comunidade, os fabricantes e o público consumidor sobre seu papel na sociedade, no meio em que vivem e no ciclo de vida dos produtos (STAHEL, 2016).

Observa-se que os conceitos e os pilares abordados não mencionam a Economia Circular, no entanto, a Economia Circular abrange todos estes conceitos de forma integrada. $\mathrm{O}$ que acontece, em grande parte das empresas, é que a Economia Circular está sendo aplicada de forma fragmentada (RITZEN; SANDSTRÖM, 2017), ou seja, apenas os conceitos que atendem aos interesses financeiros destas empresas e apresentam melhor compatibilidade técnica com seus processos operacionais já existentes. Estes conceitos, quando aplicados de forma fragmentada, servem apenas para reduzir o consumo dos recursos naturais, o que não é a solução para a limitação dos mesmos; e sim, uma forma de postergar o inevitável (ELLEN MACARTHUR FOUNDATION, 2015).

\section{Considerações finais}

Por meio deste estudo pode-se concluir que o conceito de Economia Circular é revolucionário e promissor, no entanto, ainda muito idealista. A implantação deste conceito de 
forma efetiva requer mudanças culturais, e, principalmente, uma nova visão de gestão, por parte das empresas, em virtude do comprometimento com o meio ambiente, e a preocupação com as externalidades causadas pelos processos produtivos, mesmo que estas não tenham um impacto direto nos negócios das respectivas empresas.

Em relação ao conceito de Economia Circular, foi possível constatar que o mesmo abrange diversos outros conceitos; o que torna a sua aplicação complexa e desafiadora. Portanto, é essencial a compreensão do termo Economia Circular, seus princípios e pilares, para facilitar a sua adoção, e implantação, como um modelo econômico sustentável. Analisando a literatura científica que versa sobre o referido tema, percebe-se que o conceito de Economia Circular ainda está um pouco confuso, considerando as divergências encontradas entre os diferentes autores. Sendo assim, conclui-se que o seu conceito está em fase de construção.

Ao longo do trabalho, os autores abordam diferentes pilares que sustentam a Economia Circular, e que facilitam a sua implantação. Observa-se que os pilares citados na literatura, são práticas sustentáveis que as empresas já conhecem, ou operacionalizam. O que acontece, na maioria das empresas, é que a Economia Circular está sendo aplicada de forma fragmentada, ou seja, apenas os conceitos que atendem aos interesses econômicos destas empresas, ou que apresentam melhor compatibilidade técnica com seus processos operacionais já existentes.

Além disso, a adoção de uma Economia Circular requer a mudança de valores e princípios, com a implantação das práticas sustentáveis, também, em toda a cadeia de suprimentos. Portanto, a adoção deste modelo depende não só da empresa, mas também, dos fornecedores, clientes, sociedade e poder público. A Economia Circular não irá alcançar o seu objetivo, se ocorrer de forma isolada.

Sendo assim, para que haja o maior engajamento das empresas e da sociedade, possibilitando o fechamento do círculo dos produtos e a parceria entre diferentes segmentos, se faz necessário a intervenção do governo, por meio de legislação, políticas públicas, incentivos fiscais e campanhas de conscientização.

Portanto, o estudo contribuiu com a literatura sobre a Economia Circular no Brasil, e ainda, foi possível resgatar, de diferentes artigos, de forma a detalhar o conceito e melhorar o seu entendimento como um todo, os diversos pilares, práticas sustentáveis, benefícios e as dificuldades na implantação de Economia Circular nas empresas. Como limitações do estudo, cita-se a falta de literatura sobre a situação da Economia Circular no Brasil. Como sugestão para trabalhos futuros, tem-se o estudo aprofundado das práticas sustentáveis no contexto da Economia Circular em indústrias brasileiras. 


\section{Referências}

ABRELPE, Associação Brasileira das Empresas de Limpeza Pública e Resíduos Especiais. Panorama dos Resíduos Sólidos no Brasil 2018/2019. Disponível em http://www.abrelpe.org.br/ Acesso em 23 de março de 2020.

ALBUQUERQUE, José de Lima et al. Gestão Ambiental e Responsabilidade Social: Conceitos, Ferramentas e Aplicações, São Paulo, SP: Atlas S.A, 2009, 320p

ALIGLERI, Lilian; ALIGLERI, Luiz Antonio; KRUGLIANSKAS, Isak. Cradle to Cradle: uma Análise dos Produtos Certificados para Limpeza Geral e Lavagem de Roupa. Revista Eletrônica de Gestão Organizacional. V. 14; Ed. Especial; ISSN 1679-1827; p. 88-96. May, 2016

ALVES, Isabel Joselita Barbosa da Rocha; FREITAS, Lúcia Santana. Análise comparativa das ferramentas de gestão ambiental: produção mais Limpa x Ecodesign. In.: LIRA, Waleska Silveira; CÂNDIDO, Gesinaldo Ataíde. (orgs). Gestão sustentável dos recursos naturais: uma abordagem participativa. Campina Grande: Editora Universidade Estadual da Paraíba. 325p. ISBN 9788578792824. p. 193, 2013

ASSIS, Bruno Bastos. Avaliação do Ciclo de Vida do produto como ferramenta para o desenvolvimento sustentável. 2009. 66 f. Monografia (Engenharia de Produção). Universidade Federal de Juiz de Fora. Juiz de Fora, MG, 2009

BALACEANU, Cristina; TILEA, Doina Maria; PENU, Daniel. Perspectives on Eco Economics, Circular Economy and Smart Economy. Academic Journal of Economic Studies. ISSN 2393-4913, V. 3, no 4, p. 105-109. December 2017

BOCKEN, Nancy M.P.; SHORT, S.; RANA, P.; EVANS, S. A value mapping tool for sustainable business modelling. Corporate Governance, Vol. 13, n 5, p. 482 - 497. 2013

BOTEZAT, Elena Aurelia; DODESCU, Anca Otília; VADUVA, Sebastian; FOTEA, Silvia Liana. An Exploration of Circular Economy Practices and Performance Among Romanian Producers. Journal Sustainability; v. 10, 17p. DOI: 10.3390/su10093191. September 2018

CHRTOW, Marian R.. Industrial Symbiosis: literature and taxonomy. Journals Annual Reviews. V. 25, p. 313-337. March, 2000

DIAS, Reinaldo. Gestão ambiental: responsabilidade social e sustentabilidade. 2. ed., rev. e atual. São Paulo, SP: Atlas, 2011. x, 220 p. ISBN 9788522462865

DOMINGUES, Ivan. Epistemologia das Ciências Humanas. Tomo 1: Positivismo e Hermenêutica. São Paulo: Loyola, 2004.

ELLEN MACARTHUR FOUNDATION. (2012). Towards a Circular Economy: Economic and business rationale for anaccelerated transition. January 2012. Disponível em: https://www.ellenmacarthurfoundation.org/publications

ELLEN MACARTHUR FOUNDATION. (2015). Towards a Circular Economy: Busines Rationale for an Accelerated Transiton. December, 2015. Disponível em:

https://www.ellenmacarthurfoundation.org/publications

ENGELAGE, Emanuele; BORGERT, Altair; SOUZA, Marcos Antônio. Práticas de Green Logistic: uma abordagem teórica sobre o tema. GeAS - Revista de Gestão Ambiental e Sustentabilidade. V. 5; n 3; p. 36-55; DOI: 10.5585/geas.v5i3.446; Editora Científica. Setember, 2016 
FELDMANN, Andreas; KORHONEN, Jouni; NUUR, Cali; BIRKIE, Seyoum Eshetu. Circular Economy as na essentially contested concept. Journal of Cleaner Production. Elsevier Ltda, V. 175, p. 544552, DOI 10.1016, February 2018

GHISELLINI, Patrizia; CIALANI, Catia; ULGIATI, Sergio. A reviewoncircular economy: theexpectedtransitionto a balancedinterplayofenvironmentalandeconomic systems. Journal of Cleaner Production. ElsevierLtd; V 114, p. 11-32. February, 2016.

GIANNETTI, Biagio Fernando; ALMEIDA, Cecília M. Villas Boas de; BONILLA, Sílvia H. Implementação de Eco-Tecnologias Rumo à Ecologia Industrial. Revista Administração de Empresas - RAE-eletrônica, V.2, no1. jan-jun de 2003

GNONI, Maria Grazia; MOSSA, Giorgio; MUMMOLO, Giovanni; TORNESE, Fabiana; VARRIELLO, Rossella. Circular Economy Strategies for electric and electronic equipment: a fuzzy cognitive map. Environmental Engineering and Management Journal. V. 16, p. 1807-1817, Issue 8. August 2017

GORECKI, Jaroslaw; NUNEZ-CACHO, Pedro; MORENO, Valentin Molina; IGLESIAS, Francisco. What Gets Measured, Gets Done: Development of a Circular Economy Measurement Scale for Building Industry. Journal Sustainability; V. 10, 22p.; DOI: 10.3390/su10072340. July, 2018 HABERMAS, Jürgen. La lógica de las ciencias sociales. 3. ed. Madrid, Espanha: Tecnos, 2000. $506 p$

HEIDEGGER, Martin. Introducción a la filosofía. Trad. de Manuel J. Redondo. Madri: Ediciónes Cátedra, 1999.

HEKMAN, Susan J. Hermenêutica e sociologia do conhecimento. Lisboa: Edições 70, 1990. HOUSE OF COMMONS. Growing a circular economy: Ending the throwaway society. HC-214. Londres: House of Commons/ Environmental Audit Committee. july, 2014. Disponível em: https://publications.parliament.uk/pa/cm201415/cmselect/cmenvaud/214/214.pdf

KIRCHHERR, Julian; PISCIELLI, Laura; BOUR, Ruben; SMIT, Erica Kostense; MULLER, Jennifer; TRUIJENS, Anne Huibrechtse; HEKKERT, Marko. Barriers to the Circular Economy: Evidence from the European Union (EU). Ecological Economics. Elsevier B.V.; V. 150, p. 264-272. April 2018 KORHONEN, Jouni; HONKASALO, Antero; SEPPÃLÃ, Jyri. Circular Economy: The Concept and its Limitations. Ecological Economics. Elsevier B.V; V 143, p. 37-46. July 2017.

LAHTI, Tom; WINCENT, Joakim; PARIDA, Vinit. A Definition and Theoretical Review of the Circular Economy, Value Creation, and Sustainable Business Models: Where Are We Now and Where Should Research Move in the Future? Journal Sustainability. V. 10, 19p.; DOI: 10.3390/su10082799. August, 2018

LEITÃO, Alexandra. Economia Circular: uma nova filosofia de gestão para o séc. XXI. Portuguese Journal of Finance, Management and Accounting. V. 1, no 2, p. 149-171. ISSN: 2183-3826. Setembro, 2015

MANNHEIM, Karl. Sociologia da cultura. São Paulo, Perspectiva. 1974.

MARTINS, Paulo Sérgio; ESCRIVÃO, Edmundo Filho; O meio ambiente no contexto organizacional: uma reflexão sobre a dimensão ambiental em pequenas e médias empresas. VII SEGeT - Simpósio de Excelência em Gestão e Tecnologia - 2010 Disponível em: https://www.aedb.br/seget/arquivos/artigos10/433 Pme e meio ambiente.pdf Acesso em: 17 de outubro de 2018. 
MATIVENGA, Paul; EJON, John Agwa; MBOHWA, Charles; SULTAN, Al Amin Mohamed; SHUAIB, Norshhah Aizat. Circular Economy Ownership Models: A view from South Africa Industry.

Procedia Manufacturing. 14th Global Conference on Sustainable Manufacturing. Stellenbosch, South Africa. Elsevier B.V. V. 8, p. 284-291. October 2016

MILLER, G. Tyler Jr, SPOOLMAN, Scott E. Ecologia e Sustentabilidade. 6.ed, São Paulo, SP: Cengage Learning, 2012, 295p

MOSTAGHEL, Rana; OGHAZI, Pejval. Circular Business Model Challenges and Lessons LearnedAn Industrial Perspective. Journal Sustainability. MDPI AG, DOI: 10.3390/su10030739, V. 10, no 3, March 2018

MOURA, Luiz Antônio Abdalla. Economia Ambiental Gestão de Custos e Investimentos. 3ạ ed, São Paulo, SP: Juarez de Oliveira, 2006, 254p

MOURA, Luiz Antônio Abdalla. Qualidade e Gestão Ambiental: Sustentabilidade e ISO 14.001. 6a ed, Belo Horizonte, MG, Del Rey Ltda, 2011, 418p

MURAKAMI, Fábio; SULZBACH, Alexandre; PEREIRA, Giancarlo Medeiros; BORCHARDT, Miriam; SELLITTO, Miguel Afonso. How can the Brazilian government use public policies to induce recycling and still save money? Journal of Cleaner Production. Elsevier Ltda; V. 96, p. 94 - 101. April 2014

NAIME, Roberto. Gestão de Resíduos Sólidos: Uma abordagem prática. Novo Hamburgo, RS: Feevale, 2005, 134p.

NAIME, Roberto; GARCIA, Ana Cristina de Almeida. Percepção Ambiental e Diretrizes para Compreender a questão do Meio Ambiente. Novo Hamburgo, RS: Feevale, 2004, 135p

ONU, Organização Mundial das Nações Unidas. Transformando nosso mundo: A agenda 2030 para o Desenvolvimento Sustentável. 2015. Disponível em https://nacoesunidas.org/pos2015/agenda2030/ Acesso em 24 de abril de 2018

PAZ, Fábio Josende; KIPPER, Liane Mahlmann; Sustentabilidade nas organizações: vantagens e desafios. GEPROS. Gestão da Produção, Operações e Sistemas, Bauru, Ano 11, no 2, p. 85-102; abr-jun/2016.

RAZERA, Dalton Luiz; BARAUNA, Debora; SOUZA, Silvana; TREIN, Fabiano Andre. Design para a Sustentabilidade na Economia de Materiais: uso de Resíduos no Desenvolvimento de Produtos. Mix Sustentável; Florianópolis; V. 3; p. 113-122. Outubro-Março, 2017.

REIS, Lineu Belicodos; FADIGAS, Eliane A. F. Amaral; CARVALHO, Cláudio Elias. Energia, Recursos Naturais e a Prática do Desenvolvimento Sustentável. 2. ed, Barueri, SP: Manole, 2012, 440p

RIOS, Irel Carolina; CHARNLEY, Fiona. Skills and capabilities for a sustainable and circular economy: The changing role of design. Journal of Cleaner Production. Elsevier Ltda; V 160; $p$. 109-122; October 2016

RITZEN, Sofia; SANDSTROM, Gunilla Olundh. Barriers to the Circular Economy - integration of perspective and domains. 9th CIRP IPSS Conference: Circular Perspectives on Product/ServiceSystems. Elsevier B.V.; V.64; p. 7-12. 2017

SACIROVIC, Selim; KETIN, Sonja; VIGNJEVIC, Nada. Eco-industrial zones in the context of sustainability development of urban areas. Environmental Science and Pollution Research. Springer Verlag. DOI: 10.1007/s11356-018-1390-y; p. 1-11. March 2018 
SANCHEZ, Emiliano Molina; LEYVA-DIAZ, Juan Carlos; GARCIA, Francisco Joaquin C.; MORENO, Valentin Molina. Proposal of Sustainability Indicators for the Waste Management from the Paper Industry within the Circular Economy Model. Journal Sustainability; V. 10; 17p.; DOI: 10.3390/w10081014. July 2018

SANDOVAL, Vanessa Prieto; ORMAZABAL, Marta; JACA, Carmen. Towards a consensus on the circular economy. Journal of Cleaner Production. Elsevier Ltda; V. 179, p. 605-615. December 2017

SAUVÉ, Sébastien.; BERNARD, Sophie.; SLOAN, Pamela. Environmental sciences, sustainable development and circular economy: Alternative concepts for transdisciplinary research. Environmental Development. Elsevier B.V; V. 17, p. 48-56, January 2016.

SHIGUNOV NETO, Alexandre; CAMPOS, Lucila Maria de Souza; SHIGUNOV, Tatiana.

Fundamentos da gestão ambiental. Rio de Janeiro, RJ: Ciência Moderna, 2009. xxi, 295 p. ISBN 9788573938012

SILVA, Christian Luiz da Silva; JUNIOR, Eloy Fassi Casagrande; LIMA, Isaura Alberton de; SILVA, Maclovia Corrêa da; AGUDELO, Líbia Patrícia Peralta; PIMENTA, Rosângela Borges. Inovação e Sustentabilidade. Curitiba, PR: Aymará Educação, 2012. Disponível em:

http://repositorio.utfpr.edu.br/ispui/bitstream/1/2066/1/inovacaosustentabilidade.pdf Acesso em: 25 de abr de 2018

SILVEIRA, Michele Plentz; ALVES, Juliano Nunes. Sistemas de Gestão Ambiental: benefícios e dificuldades. XVII Seminário Interinstitucional de Ensino, Pesquisa e Extensão. UniCruz Universidade de Cruz Alta. Cruz Alta/RS. Novembro 2012. Disponível em:

https://home.unicruz.edu.br/seminario/downloads/anais/ccsa/sistema\%20de\%20gestao\%20a mbiental\%20beneficios\%20e\%20dificuldades.pdf Acesso em: 17 de outubro de 2018.

SMOL, Marzena; KULCZYCKA, Joanna; AVDIUSHCHENKO, Anna. Circular Economy indicators in relation to ecoinnovation in European regions. Springer Verlag. V. 19, n 3, p. 669-678.

DOI 10.1007/s10098-016-1323-8. January 2017

SPOSATO, Paola; PREKA, Rovena; CAPPELLARO, Francesca; CUTAIA, Laura. Sharing Economy and circular economy: how technology and collaborative consumption innovations boost closing the loop strategies. Environmental Engineering and Management Journal. Gheorghe Asachi Technical University of Lasi. V. 16; no 8; p. 1797-1806. August 2017

STAHEL, Walter R. Circular economy: a new relationship whit our goods and material swould save resources and energy and create local Jobs. Nature Publishing Group; Macmillan Publishers Limited. V. 531; Issue 7595; p. 435 - 438. March 2016.

TANTAU, Adrian Dumitru; MAASSEN, Maria Alexandra; FRATILA, Laurentiu. Models for Analyzing the Dependencies between Indicators for a Circular Economy in the Europe Union. Journal Sustainability; V. 10, 13p. DOI: 10.3390/su10072141. June 2018

URBINATI; Andrea; CHIARONI, Davide; CHIESA, Vittorio. Towards a new taxonomy of circular economy business models. Journal of Cleaner Production. Elsevier Ltda; V. 168, p. 487 - 498. September 2017

VALENTURF, Anne P.M; PURNELL, Phil; TREGENT, Mike; FERGUSON, John; HOLMES, Alan. CoProducing a Vision and Approach for the Transition towards a Circular Economy: Perspectives from Government Partners. Journal Sustainability; V. 10, 20p. DOI: 10.3390/su10051401. May 2018 
VELIS, Costas A.; VRANCKEN, Karl C.. Which material owner ship and responsability in a circular economy? The Journal of the International Solid Wastes and Public Cleansing Association.

Sage Publications. V. 33, no 9, p. 773-774, ISSN: 1096-3669. September 2015

WASTLING, Thomas; CHARNLEY, Fiona; MORENO, Mariale. Design for Circular Behaviour: Considering Users in a Circular Economy. Journal Sustainability; V. 10, 22p.; DOI:

10.3390/su10061743. May, 2018

WEBER, M. Ensaios de sociologia. Rio de Janeiro: Zahar Editores, 1979. 